\title{
BOAS PRÁTICAS EM MONITORIZAÇÃO ELETROCARDIOGRÁFICA: REVISÃO INTEGRATIVA
}

\section{Wellington Pereira da Silva*, Angela Carolina Brandão De Souza Giusti, Marilia Estevam Cornélio,}

\author{
Thaís Moreira São João, Maria Cecília Gallani.
}

\section{Resumo}

Objetivo: identificar as barreiras ou problemas enfrentados pelos enfermeiros para realizar a monitorização cardíaca de acordo com as recomendações atuais e apontar as intervenções realizadas para solução dos problemas identificados. Método: Foi relaizada uma revisão integrativa da literatura. Para a seleção dos estudos, utilizou-se as bases de dados PubMed, Scopus e Web of Science. Resultados: foram incluídos 20 artigos, sendo as principais barreiras para a realização da monitorização cardíaca adequada o desconhecimento sobre recomendações para a correta monitorização eletrocardiográfica e a dificuldade de interpretação do ritmo cardíaco. Estratégias de educação continuada e personalização dos parâmetros dos monitores têm demonstrado serem eficazes para que as boas práticas na monitorização cardíaca sejam implementadas nas instituições hospitalares.

\section{Palavras-chave:}

Eletrocardiografia, Enfermagem, Monitorização

\section{Introdução}

Atualmente, já é possível identificar, por meio de monitores cardíacos cada vez mais avançados, diversos parâmetros cardíacos, como o ritmo, arritmias complexas, alterações eletrocardiográficas relacionadas

à isquemia do miocárdio e prolongamento do intervalo QT. Isso possibilita o diagnóstico precoce e o tratamento imediato, por exemplo, de doenças como Infarto Agudo do Miocárdio (IAM) e distúrbios elétricos, como as fibrilações cardíacas $^{(1,2)}$.

a American Heart Association (AHA) lançou, em 2004, uma recomendação com padrões para a prática do monitoramento eletrocardiográfico para o ambiente hospitalar, com atualização mais recente em 2017, temos no documento indicações para a monitorização contínua do ECG específicas para os sinais e sintomas apresentados por cada paciente ${ }^{(1,2)}$.

Mesmo havendo evidências de que a habilidade de leitura e interpretação dos dados oferecidos pelos monitores cardíacos seja fundamental para a assistência ao paciente monitorizado, evidencia-se um despreparo de enfermeiros para realizar uma monitorização adequadal(3).

O objetivo do estudo foi identificar as barreiras ou problemas enfrentados pelos enfermeiros para realizar a monitorização cardíaca de acordo com as recomendações atuais e apontar as intervenções realizadas para solução dos problemas identificados.

\section{Resultados e Discussão}

Realizou-se uma revisão integrativa(4), que contemplou as seguintes etapas: 1 . Estabelecimento da questão da pesquisa; 2 . Definição dos critérios de inclusão e exclusão. 3. Extração de tados; 4. Avaliação dos estudos incluídos; 5 . Interpretação dos dos dados e

sintese do conhecimento. Para a seleção dos estudos, utilizou-se as bases de dados PubMed, Scopus e Web of Science.

Foram selecinados 20 artigos que estão distribuídos entre os seguintes anos: 2014 (seis), 2015 (quatro), 2016 (cinco), 2017 (dois) e 2018 (três). Houve predomínio dos estudos encontrados na base de dados PubMed (19). Todos os estudos selecionados estavam em inglês e tem origem em sete diferentes regiões do mundo, sendo 14 nos Estados Unidos da América, um na Austrália, um no Brasil, um na Espanha, um na Noruega, um no Reino Unido e um na Suécia. Quanto ao tipo de estudo, dez apresentavam abordagem quantitativa, quatro qualitativa, dois estudos eram qualiquantitativos e quatro se tratavam de revisões. As principais barreiras para a realização da monitorização cardíaca adequada 0 desconhecimento sobre recomendações para a correta monitorização eletrocardiográfica; a dificuldade de interpretação do ritmo cardíaco; o excesso de alarmes e sinais sonoros emitidos pelos monitores; a colocação incorreta dos eletrodos; falta do preparo ou preparo inadequado da pele; e dificuldade em reter o conhecimento após intervenções educacionais.

\section{Conclusões}

Apesar das barreiras encontradas, estratégias de educação continuada, estabelecimento de protocolos institucionais e personalização dos parâmetros dos monitores têm demonstrado serem eficazes para que as boas práticas na monitorização cardíaca sejam implementadas nas instituições hospitalares.

\section{Financiamento}

* Bolsista de Iniciação Científica pelo Conselho Nacional de Desenvolvimento Científico e Tecnológico (CNPq).

1- Sandau KE, Funk M, Auerbach A, Barsness GW, Blum K, Cvach M, et al Update to practice standards for electrocardiographic monitoring in hospital. A scientifc statement from the American Heart Association. Endorsed by the American College of Cardiology, American Association of Critical-Care Nurses, and Pediatric and Congenital Electrophysiology Society

2- Drew BJ, Califf RM, Funk M, Kaufman ES, Krucoff MW, Laks MM Macfarlane PW, Sommargren C, Swiryn S, Van Hare GF. Practice standards for electrocardiographic monitoring in hospital settings: an American Heart Association scientific statement.

3- Pickham D, Shinn JA, Chan GK, Funk M, Drew BJ. Quasi-experimental study to improve nurses' QT-interval monitoring: results of QTIP study. Am J Crit Care [Internet]. 2012;21(3):195-200

4- Mendes KDS, Silveira RCP, Galvão CM. Revisão integrativa: método de pesquisa para a incorporação de evidências na saúde e na enfermagem. Texto \& Contexto - Enfermagem [Internet]. 2008;17(4):758-64 